\title{
Identification of the novel protein FAM172A, and its up-regulation by high glucose in human aortic smooth muscle cells
}

\author{
LIANXI LI $^{1 *}$, XUEHONG DONG ${ }^{2 *}$, MAN CHEONG LEONG $^{2}$, WENBAI ZHOU ${ }^{2}$, ZHIHONG YANG $^{2}$, \\ FENGLING CHEN $^{2}$, YUQIAN BAO ${ }^{1}$, WEIPING JIA ${ }^{1}$ and RENMING HU ${ }^{2}$ \\ ${ }^{1}$ Department of Endocrinology and Metabolism, Shanghai Clinical Center for Diabetes, Shanghai Jiao Tong University \\ Affiliated Sixth People's Hospital, Shanghai Diabetes Institute, Shanghai Key Laboratory of Diabetes Mellitus, \\ Shanghai 200233; ${ }^{2}$ Department of Endocrinology and Metabolism, Huashan Hospital, \\ Institute of Endocrinology and Diabeteology, Fudan University, Shanghai 200040, P.R. China
}

Received May 14, 2010; Accepted July 12, 2010

DOI: 10.3892/ijmm_00000489

\begin{abstract}
The family with sequence similarity 172, member A (FAM172A) is a hypothetical protein. We recently cloned the FAM172A gene from normal human aortic tissues. In a previous study we also showed that the FAM172A gene was up-regulated by high glucose levels in macrophages. In the present study, we further identified the FAM172A protein at the level of translation and studied the effects of high glucose levels on its expression in human aortic smooth muscle cells. The FAM172A gene was subcloned into the eukaryotic expression vectors, PDC315 and pEGFP-N2. The cloned sequence shows an open reading frame of 1251 nucleotides encoding a protein of 416 amino acids. We further expressed the recombinant FAM172A protein and generated rabbit anti-human FAM172A polyclonal antibodies. The FAM172A protein was identified for the first time at the translation level by Western blot analysis. Western blotting also demonstrated that the FAM172A protein could be detected in human aortic endothelial, human aortic smooth muscle cells and THP-1-derived macrophages, the highest expression being observed in the human aortic smooth muscle cells. By a combination of bioinformatics and
\end{abstract}

Correspondence to: Dr Weiping Jia, Department of Endocrinology and Metabolism, Shanghai Clinical Center for Diabetes, Shanghai Jiao Tong University Affiliated Sixth People's Hospital, Shanghai Diabetes Institute, Shanghai Key Laboratory of Diabetes Mellitus, 600 Yishan Road, Shanghai 200233, P.R. China

E-mail:wpjia@sjtu.edu.cn

Dr Renming Hu, Department of Endocrinology and Metabolism, Huashan Hospital, Institute of Endocrinology and Diabeteology, Fudan University, 12 Middle Urumqi Road, Shanghai 200040, P.R. China

E-mail: renming_hu@hotmail.com

${ }^{*}$ Contributed equally

Key words: diabetic macroangiopathy, vascular smooth muscle cell, family with sequence similarity 172 member A, glucose confocal laser scanning microscopy, we found that the FAM172A protein in HEK293 cells, was mainly located in the nucleus, and that there was an Arb2 conserved domain in the FAM172A protein sequence. We also presented evidence that the FAM172 protein expression in human aortic smooth muscle cells was up-regulated by high glucose levels in a concentration-dependent and time-course manner. We speculated that as a novel protein, FAM172A could be involved in the pathogenesis of high glucose-induced vascular damage.

\section{Introduction}

The chronic complications of diabetes are generally divided into microvascular and macrovascular complications. The diabetic macrovascular disease or atherosclerosis and its complications, are the leading causes of morbidity and mortality in patients with type 2 diabetes mellitus $(1,2)$. The histopathology of atherosclerosis in patients with diabetes is similar to that in patients without diabetes. However, compared to non-diabetic patients, atherosclerotic vascular damage in diabetic patients is more diffuse, more complicated and with clinically worse outcomes (3). Although insulin resistance, hypertension, dyslipidemia and other risk factors certainly contribute to the progression of diabetic macroangiopathy (3-8), the exact mechanisms of these pathological processes remain to be elucidated.

Chronic hyperglycemia is a hallmark characteristic of diabetes and exerts its adverse effects on the cardiovascular system in many ways (7). There is universal agreement that hyperglycemia is a powerful and independent risk factor for diabetic cardiovascular complications (9-13). Therefore, hyperglycemia plays a crucial role in the pathogenesis of diabetic macrovascular complications.

Glucose is one of the most important factors by which the expressions of genes in vascular or other cells are regulated. We, as well as others, have previously suggested that elevated glucose levels can cause altered gene expression in vascular and islets cells (14-17). Many glucose-induced genes have been identified in vascular cells. Nicolson identified new 
high glucose-induced genes in bovine aortic smooth muscle cells by mRNA differential display (18). He also reported that these genes were differentially regulated by high $(22 \mathrm{mM})$ compared to low glucose $(5.5 \mathrm{mM})$ concentrations. In another study, high glucose-induced gene expression profiles were established in THP-1 monocytes, as well as a variety of proinflammatory cytokines, adhesion molecules, chemokine receptors and integrins. Also, chemokines relevant to the pathogenesis of diabetes complications were induced by high glucose levels (15). It is well known that elevated glucose levels can stimulate diacylglycerol accumulation and activate protein kinase $\mathrm{C}$ in vascular cells, which are known modulators of gene expression. Therefore, the high glucose-induced gene expression in vascular cells could be one of the mechanisms involved in the pathogenesis of diabetic macrovascular complications.

In our previous study, we identified the novel gene [family with sequence similarity 172, member A (FAM172A, also named C5orf21)] in normal human aortic tissue. This gene was also regulated by high glucose levels in human THP-1derived macrophages (19). The FAM172A protein is a hypothetical protein and little is known about it. It is even unclear whether or not this protein is present at the level of translation.

In the present study, we further confirmed the presence of the FAM172A gene at the level of protein translation. We also determined that the FAM172A protein in HEK293 cells was mainly located in the nucleus. Finally, we presented evidence that high glucose levels up-regulated the expression of the FAM172A protein in human aortic smooth muscle cells in a concentration-dependent and a time-course manner.

\section{Materials and methods}

Bioinformatics analysis. The database of the National Center for Biotechnology Information (NCBI) was used for the sequence analysis and for the search of the FAM172A conserved domain. Online software CELLO 2.5 (http:// cello.life.nctu.edu.tw/) was used to predict the subcellular localization of the human FAM172A protein (20).

Cell culture. Human aortic smooth muscle cells (Cascade Biologics) were cultured in medium 213 supplemented with Smooth Muscle Growth Supplement (Cascade Biologics) in an atmosphere of $95 \%$ air and $5 \% \mathrm{CO}_{2}$ at $37^{\circ} \mathrm{C}$ in culture flasks. Human aortic endothelial cells (Cascade Biologics) were cultivated in medium 200 containing Low Serum Growth Supplement (Cascade Biologics) at $37^{\circ} \mathrm{C}$ with $5 \%$ $\mathrm{CO}_{2}$. Cells from the human monocytic cell line, THP-1 (American Type Culture Collection, ATCC), were grown in RPMI-1640 (Gibco) containing 10\% fetal bovine serum (HiClone) at $37^{\circ} \mathrm{C}$ with $5 \% \mathrm{CO}_{2}$. The differentiation of the THP-1 cells to the macrophages was induced with $160 \mathrm{nM}$ 12-O-tetradecanoyl-phorbol-13-acetate (Cell Signaling Technology) in normal growth medium for $48 \mathrm{~h}$. The HEK293 cells (ATCC) were maintained in DMEM (Gibco) containing $10 \%$ fetal bovine serum (HiClone) at $37^{\circ} \mathrm{C}$ with $5 \% \mathrm{CO}_{2}$.

Cell treatment. The human aortic smooth muscle cells were treated with different concentrations of glucose $(5.5,15$ and
$25 \mathrm{mmol} / \mathrm{l}$ ) for $48 \mathrm{~h}$. The cell cultures that were exposed to $25 \mathrm{mmol} / \mathrm{l}$ mannitol were used as the osmolar control. In some experiments, the cells were treated with $25 \mathrm{mmol} / \mathrm{l}$ glucose for $1,48,72$ or $96 \mathrm{~h}$, respectively.

Prokaryotic expression and antibody generation. Two oligonucleotide primers were designed to amplify the truncated open reading frame (ORF) sequences of the human FAM172A gene from $56 \mathrm{bp}$ downstream of the initiator ATG to the stop codon. The oligonucleotide sequences were as follows: 5'CAAGAATTCCAAATCCAGCAGGGAGGTCC-3' and 5'GCGAAGCTTGTTACAGCTCTTCGTGCTTGA-3' (the EcoRI and HindIII sequences are underlined). Using Phusion High-Fidelity DNA Polymerase (New England Biolabs, Inc.), a polymerase chain reaction (PCR) was performed on the Pdrive-FAM172A plasmid, which was constructed in our previous study (19). The PCR conditions were $98^{\circ} \mathrm{C}$ for $30 \mathrm{sec}$, followed by 33 cycles at $98^{\circ} \mathrm{C}$ for $10 \mathrm{sec}, 68^{\circ} \mathrm{C}$ for $30 \mathrm{sec}$ and $72^{\circ} \mathrm{C}$ for $45 \mathrm{sec}$. The resulting PCR products were cut with EcoRI and HindIII and subsequently subcloned into the pET28a prokaryotic expression vector (Novagen). The construct of pET28a-FAM172A was verified by sequencing analysis. The recombinant pET28a-FAM172A vector was transformed into the Escherichia (E.) coli strain, DH5 $\alpha$, to express the His-tagged fusion protein. The fusion protein expression was induced with $1 \mathrm{mM}$ isopropyl- $\beta$-D-thiogalactopyranoside (IPTG) for $3 \mathrm{~h}$ at $37^{\circ} \mathrm{C}$. The recombinant protein was purified according to the protocol recommended by the manufacturer of the His-tag kit (Novagen). The purified recombinant FAM172A protein was emulsified in complete Freund's adjuvant. Two New Zealand white rabbits were subcutaneously immunized 3 times with $100 \mu \mathrm{g}$ purified FAM172A protein. The serum was collected 1 month after the third immunization, and then the antibody titer against the recombinant protein was tested by ELISA. The rabbit antiserum was diluted at 1:5000 for Western blotting.

Eukaryotic expression and transfection. The pDriveFAM172A plasmid was used as the template for the amplification of the human FAM172A ORF. In order to construct pEGFP-N2-FAM172 plasmid, the human FAM172A ORF was amplified with primers containing EcoRI (5'-CGCGAATTCATGTCTATTTCCTTGAGCTC3') and BamHI (5'-GTGGATCCCATGTCTTTCCTCTTG TT-3') sites and subcloned into the EcoRI/BamHI sites of the pEGFP-N2 eukaryotic expression vector (Clontech). The PCR conditions were the same as those described above for the pET28a-FAM172A plasmid construction. In order to construct the PDC315-FAM172 plasmid, 2 primers were designed to amplify the human FAM172A ORF. The primer sequences were 5'-CGCGAATTCATGTCTATTTCCT TGAGCTC-3' and 5'-GAAGGATCCTTACAGCTCTTC GTGCTTGA-3' (the EcoR I and BamHI sequences are underlined). The PCR conditions were the same as those described above. The resulting PCR products were cut with EcoRI and BamHI, and then subcloned into the PDC315 eukaryotic expression plasmid (Microbix Biosystems) for the construction of the recombinant PDC315-FAM172A plasmid. All the plasmids were transformed into the E. coli strain, and then isolated and purified using the Plasmid Maxi Kit 
(Qiagen). The HEK293 cells were transiently transfected with the appropriate PDC315- or PDC315-FAM172A plasmids using Lipofectamine 2000 (Invitrogen) according to the manufacturer's instructions.

Fluorescence microscopy. The HEK293 cells were transiently transfected with the appropriate pEGFP-N2-FAM172A constructs using Lipofectamine 2000 according to the instruction manual. At $48 \mathrm{~h}$ after transfection, the HEK293 cells were trypsinized and transferred to coverslips in 24-well plates. The cells were rinsed for 1 min with PHEM buffer and permeabilized for 1 min with PHEM plus $0.1 \%$ Triton $\mathrm{X}-100$. The cells were fixed in freshly made $4 \%$ paraformaldehyde, and rinsed 3 times in PBS. The coverslips were blocked with $0.05 \%$ Tween-20 in PBS (TPBS) with $1 \%$ bovine serum albumin. The nuclei of the cells were then stained with DAPI. The GFP and DAPI localization was visualized by direct fluorescence with a confocal microscopy system (Zeiss).

Western blot analysis. The FAM172A protein expression was detected in the total cell lysates with rabbit polyclonal antiserum. The protein samples were subjected to SDSpolyacrylamide (5\% stacking and 10\% running gel) electrophoresis followed by electroblotting onto nitrocellulose membranes. The membranes were blocked using 5\% non-fat dry milk in Tris-buffered saline with $0.05 \%$ Tween-20 and incubated with rabbit polyclonal antiserum at a dilution of 1:5000. The membranes were then washed and incubated with a secondary anti-rabbit IgG peroxidase-conjugated antibody (Cell Signaling Technology) diluted at 1:1000 in the same buffer for $1 \mathrm{~h}$. The normalization was done with mouse monoclonal anti-actin antibody (Santa Cruz). The protein bands were visualized by chemiluminescence with an ECL luminescence kit (Pierce) and exposed to X-ray film. The protein band intensities were quantitated with scanning densitometry.

\section{Results}

Bioinformatics analysis of FAM172A. The human FAM172A gene was mapped on chromosome $5 \mathrm{q} 15$. Its ORF contained $1251 \mathrm{bp}$, encoding a protein of 416 amino acids. Fig. 1A demonstrates the nucleotide and amino acid sequences of the human FAM172A ORF. Online software-based prediction analysis showed that there was an Arb2 conserved domain in the FAM172A protein sequence (as shown in Fig. 1A and B). Online software CELLO 2.5 prediction analysis showed that the human FAM172A protein was located in the nucleus and/or cytoplasm (Fig. 1C). After a BLAST search of the nucleotide sequence of the human FAM172A gene was performed in the NCBI database, we found that the FAM172A gene was conserved in the chimpanzee, dog, cow, mouse, rat, chicken, zebrafish, fruit fly, mosquito and C. elegans.

Purification of recombinant FAM172A protein and generation of anti-FAM172A polyclonal antibody. After the transfection of the recombinant pET28a-FAM172A vector into $E$. coli and IPTG induction, a prominent band of $\sim 48 \mathrm{kDa}$, which agreed with the predicted size of the recombinant protein, was detected by SDS-PAGE (Fig. 2A). The fusion protein was purified from the E. coli lysates by the His-Tag purification kit.

After 3 immunizations of a rabbit with the recombinant FAM172A protein, the rabbit serum containing the antiFAM172A polyclonal antibody was collected, and then the high titer antibody activity was tested by using ELISA. Recombinant FAM172A protein (100 ng) was loaded onto a $10 \%$ SDS-PAGE gel and detected with the rabbit antiserum as the primary antibody. The results of Western blotting showed that the polyclonal antibody against the FAM172A protein, was specific and validated (Fig. 2B).

Identification of FAM172A protein at translation level and detection in vascular cells by Western blotting. In order to determine if the FAM172A was expressed at the level of translation, after the transfection of the recombinant PDC315FAM172A plasmid into the HEK293 cells, a markedly enhanced band $48 \mathrm{kDa}$ was observed by Western blotting (as shown in Fig. 3A). The result proved for the first time that the FAM172A gene was expressed at the level of translation. Western blot analysis was also used to investigate whether or not the FAM172 protein was detectable in vascular cells including human aortic endothelial, human aortic smooth muscle cells and THP-1-derived macrophages. Western blotting demonstrated that the FAM172A protein was detectable in human aortic endothelial, human aortic smooth muscle cells and macrophages, and the highest expression was observed in human aortic smooth muscle cells (Fig. 3B).

Subcellular localization of FAM172A protein. In order to determine the subcellular localization of the FAM172A protein, the recombinant pEGFP-N2-FAM172A construct containing the FAM172A ORF was transiently transfected into human HEK293 cells. After transfection, DNA was stained by DAPI (blue). Finally, the transfected HEK293 cells were examined under a fluorescent microscope. The transfection of the cells with the pEGFP-N2-FAM172A construct showed that the green fluorescent product, FAM172A-GFP, was strong in the cell nucleus with weak green fluorescent product in the cytoplasm (Fig. 4A and B). The convergence of the 2 images gave a yellow fluorescent pattern, which confirmed the co-location of the FAM172AGFP fusion protein and the cell nuclei (Fig. 4C).

Regulation of FAM172A protein expression by glucose. Given that the expression of FAM172A was very high in human aortic smooth muscle cells, we chose these cells in order to study whether the FAM172A protein expression was regulated by glucose levels. Human aortic smooth muscle cells were cultured with various concentrations of glucose $(5.5,15$ and $25 \mathrm{mmol} / \mathrm{l})$ for $48 \mathrm{~h}$, or with high glucose concentrations $(25 \mathrm{mmol} / \mathrm{l})$ for various time periods $(1,48$, 72 or 96 h). Cells cultured with $25 \mathrm{mM}$ mannitol were used as the osmolar control. As shown in Fig. 5B, the FAM172A protein expression had significant differences between the low and high glucose concentrations in the 48-h culture time. Compared to the $5.5 \mathrm{mM}$, the $15 \mathrm{mM}$ and $25 \mathrm{mM}$ glucose concentrations markedly increased the expression of the FAM172A protein in human aortic smooth muscle cells. 
A

01

ATGTCTATTTCCTTGAGCTCTTTAATTTTGTTGCCAATTTGGATAAACATGGCACAAATC

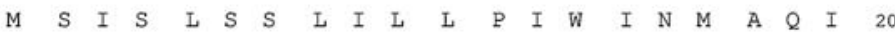

61

CAGCAGGGAGGTCCAGATGAAAAAGAAAAGACTACCGCACTGAAAGATTTATTATCTAGG

$\begin{array}{llllllllllllllllllllll}Q & Q & G & G & P & D & E & K & E & K & T & T & A & L & K & D & L & L & S & R & 40\end{array}$

121

ATAGATTTGGATGAACTAATGAAAAAAGATGAACCGCCTCTTGATTTTCCTGATACCCTG

$\begin{array}{lllllllllllllllllllll}I & D & L & D & E & L & M & K & K & D & E & P & P & L & D & F & P & D & T & L & 60\end{array}$

181 GAAGGATTTGAATATGCTTTTAATGAAAAGGGACAGTTAAGACACATAAAAACTGGGGAA

$\begin{array}{llllllllllllllllllllll}\text { E } & G & F & E & Y & A & F & N & E & K & G & Q & L & R & H & I & K & T & G & E & 80\end{array}$

241 CCATTTGTTTTTAACTACCGGGAAGATTTACACAGATGGAACCAGAAAAGATACGAGGCT

$\begin{array}{llllllllllllllllllllllll}P & F & V & F & N & Y & R & E & D & L & H & R & W & N & Q & K & R & Y & E & A & 100\end{array}$

301

CTAGGAGAGATCATCACGAAGTATGTATATGAGCTCCTGGAAAAGGATTGTAATTTGAAA

$\begin{array}{lllllllllllllllllllll}\text { L } & G & E & I & I & T & K & \text { Y } & \text { V } & \text { Y } & \text { E } & \text { L } & \text { L } & \text { E } & \text { K } & \text { D } & \text { C } & \text { N } & \text { L } & \text { K } & 120\end{array}$

361 AAAGTATCTATTCCAGTAGATGCCACTGAGAGTGAACCAAAGAGTTTTATCTTTATGAGT

$\begin{array}{llllllllllllllllllllll}K & V & S & I & P & V & D & A & T & E & S & E & P & K & S & F & I & F & M & S & 140\end{array}$

421 GAGGATGCTTTGACAAATCCACAGAAACTGATGGTTTTAATTCATGGTAGTGGTGTTGTC

$\begin{array}{llllllllllllllllllllll}\text { E } & \text { D } & \text { A } & \text { L } & \text { T } & \text { N } & \text { P } & \text { Q } & \text { K } & \text { L } & \text { M } & \text { V } & \text { L } & \text { I } & \text { H } & \text { G } & \text { S } & \text { G } & \text { V } & \text { V } & 160\end{array}$

481 AGGGCAGGGCAGTGGGCTAGAAGACTTATTATAAATGAAGATCTGGACAGTGGCACACAG

$\begin{array}{llllllllllllllllllllll}\mathrm{R} & \mathrm{A} & \mathrm{G} & \mathrm{Q} & \mathrm{W} & \mathrm{A} & \mathrm{R} & \mathrm{R} & \mathrm{L} & \mathrm{I} & \mathrm{I} & \mathrm{N} & \mathrm{E} & \mathrm{D} & \mathrm{L} & \mathrm{D} & \mathrm{S} & \mathrm{G} & \mathrm{T} & \mathrm{Q} & 180\end{array}$

541 ATACCGTTTATTAAAAGAGCTGTGGCTGAAGGATATGGAGTAATAGTACTAAATCCCAAT

$\begin{array}{llllllllllllllllllllllll}I & P & F & I & K & R & A & V & A & E & G & Y & G & V & I & V & \text { L } & \text { N } & \text { P } & \text { N } & 200\end{array}$

601 GAAAACTATATTGAAGTAGAAAAGCCGAAGATACACGTACAGTCATCATCTGATAGTTCA

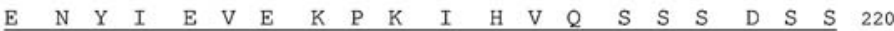

661 GATGAaCCAGCAGAAAAACGGGAAAGAAAAGATAAAGTTTCTAAAGAAACAAAGAAGCGA

$\begin{array}{llllllllllllllllllllll}D & E & P & A & E & K & R & E & R & K & D & K & V & S & K & E & T & K & K & R & 240\end{array}$

721 CGTGATTTCTATGAGAAGTATCGTAACCCCCAAAGAGAAAAAGAAATGATGCAATTGTAT

$\begin{array}{llllllllllllllllllllll}R & D & F & Y & E & K & Y & R & N & P & Q & R & E & K & E & M & M & Q & L & Y & 260\end{array}$

781 ATCAGAGAAAATGGTTCTCCTGAAGAACATGCAATCTATGTTTGGGATCATTTCATAGCT

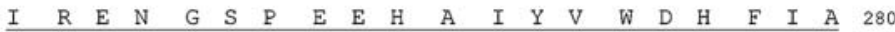

841 CAGGCTGCTGCTGAGAATGTGTTTTTCGTTGCTCACAGCTATGGAGGACTTGCTTTTGTT

$\begin{array}{lllllllllllllllllllll}\text { Q } & \text { A } & \text { A } & \text { A } & \text { E } & \text { N } & \text { V } & \text { F } & \text { F } & \text { V } & \text { A } & \text { H } & \text { S } & \text { Y } & \text { G } & \text { G } & \text { L } & \text { A } & \text { F } & \text { V } & 300\end{array}$

901 GAACTGATGATTCAACGAGAAGCCGATGTAAAAAATAAGGTAACTGCTGTGGCATTGACA

$\begin{array}{llllllllllllllllllllll}\text { E } & \text { L } & M & \text { I } & Q & \text { R } & \text { E } & \text { A } & \text { D } & \text { V } & \text { K } & \text { N } & \text { K } & \text { V } & \text { T } & \text { A } & \text { V } & \text { A } & \text { L } & \text { T } & 320\end{array}$

961 GACTCTGTTCACAATGTGTGGCATCAAGAAGCTGGCAAAACGATTCGAGAATGGATGAGA

$\begin{array}{llllllllllllllllllllll}\mathrm{D} & \mathrm{S} & \mathrm{V} & \mathrm{H} & \mathrm{N} & \mathrm{V} & \mathrm{W} & \mathrm{H} & \mathrm{Q} & \mathrm{E} & \mathrm{A} & \mathrm{G} & \mathrm{K} & \mathrm{T} & \mathrm{I} & \mathrm{R} & \mathrm{E} & \mathrm{W} & \mathrm{M} & \mathrm{R} & 340\end{array}$

1021 GAGAACTGTTGTAATTGGGTCTCTAGCTCAGAACCATTAGACACATCAGTGGAGTCCATG $\begin{array}{llllllllllllllllllllll}\text { E } & N & C & C & N & W & V & S & S & S & E & \text { P } & \text { L } & \text { D } & \text { T } & \text { S } & \text { V } & \text { E } & \text { S } & \text { M } & 360\end{array}$

1081 CTACCTGATTGCCCCCGGGTCTCAGCAGGCACCGACCGTCACGAGCTAACTTCCTGGAAG

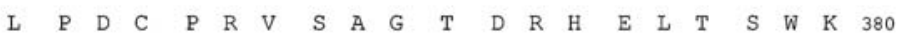

1141 AGCTTTCCGTCTATTTTCAAATTCTTTACCGAAGCCTCAGAGGCCAAGACCAGCTCCCTG $\begin{array}{llllllllllllllllllllll}S & F & P & S & I & F & K & F & F & T & E & A & S & E & A & K & T & S & S & L & 400\end{array}$

1201 AAGCCGGCTGTGACGCGCCGCTCCCACCGCATCAAGCACGAAGAGCTGTAA.

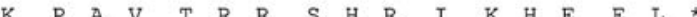
416

Figure 1. Bioinformatics analysis of human FAM172A. (A) The nucleotide and amino acid sequences of the human FAM172A ORF. The ORF of human FAM172A contained $1251 \mathrm{bp}$, encoding a protein of 416 amino acids. The amino acid sequences of the FAM172A ORF are numbered starting with the putative initiating methionine. The sequences of the predicted Arb2 conserved domain are underlined. 
B

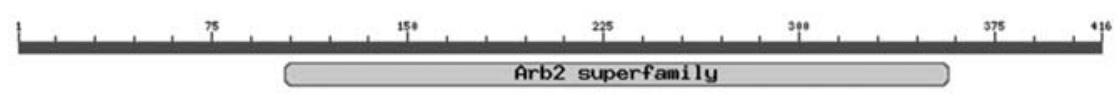

C

SeqID: FAM172A

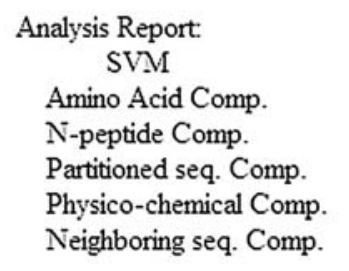

CELLO Prediction:

$\begin{array}{cc}\text { LOCALIZATION } & \text { RELIABILITY } \\ \text { Cytoplasmic } & 0.525 \\ \text { Cytoplasmic } & 0.400 \\ \text { Cytoplasmic } & 0.486 \\ \text { Cytoplasmic } & 0.423 \\ \text { Cytoplasmic } & 0.421\end{array}$

\begin{tabular}{|ll|}
\hline Cytoplasmic & $2.255 *$ \\
Nuclear & $1.681 *$ \\
\hline Criloroplast & 0.218 \\
Extracellular & 0.221 \\
Mitochondrial & 0.195 \\
Golgi & 0.080 \\
Plasma membrane & 0.073 \\
ER & 0.073 \\
Peroxisomal & 0.057 \\
Lysosomal & 0.017 \\
Cytoskeletal & 0.016 \\
Vacuole & 0.013
\end{tabular}

Figure 1. (B) The predicted Arb2 conserved domain of the human FAM172A protein. Using CD-Search, an Arb2 conserved domain was detected in the human FAM172A protein sequence. (C) The predicted subcellular localization of the human FAM172A protein. CELLO 2.5 online software prediction analysis showed that the human FAM172A protein was located in the nucleus and/or cytoplasm.

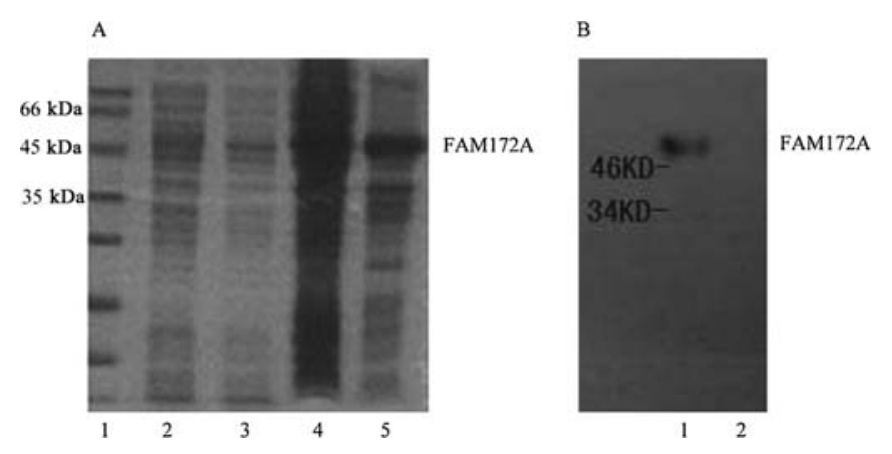

Figure 2. Expression of the recombinant FAM172A protein and the generation of the anti-FAM172A polyclonal antibody. (A) Expression of the HisFAM172A fusion protein. The lysates were analyzed by $10 \%$ SDS-PAGE, followed by Coomassie Brilliant Blue R250 staining. Lane 1, protein marker; 2, total protein before IPTG induction; 3 , total protein after IPTG induction; 4 supernatant of ultrasound-treated E. coli after IPTG induction; 5, precipitation under ultrasound-treated E. coli after IPTG induction. (B) Generation of rabbit polyclonal anti-serum against the recombinant FAM172A protein. Western blotting was used to detect the anti-FAM172A polyclonal antibody. The recombinant FAM172A protein $(100 \mathrm{ng})$ was loaded and the dilution of the polyclonal anti-serum was 1:5,000. Lane 1, FAM172A-His fusion protein; 2, bovine serum albumin as the control.

Mannitol (25 mM) had no effect on the expression of the FAM172A protein. Western blotting also showed that the expression of the FAM172A protein was augmented with high glucose concentrations in the 48-, 72- and 96-h culture times, respectively, when compared to the control group (Fig. 5A).

\section{Discussion}

Chronic hyperglycemia is now recognized as a major pathogenic factor contributing to the development and progression of diabetic macrovascular disease, including coronary artery disease, stroke, and peripheral arterial disease. However, the exact pathogenesis of diabetic macroangiopathy is not yet well understood. At present, there are at least 5 hypotheses explaining the potential mechanisms of hyperglycemiamediated vascular damage: i) Increased polyol pathway flux, ii) increased formation of advanced glycation end products, iii) activation of protein kinase $\mathrm{C}$ ( $\mathrm{PKC}$ ) isoforms, iv) increased hexosamine pathway flux, and v) increased oxidative stress $(21,22)$. In 2005, Brownlee put forward a hypothesis linking these pathogenic mechanisms, explaining the overproduction of superoxide by the mitochondrial electron transport chain (23). For example, hyperglycemiainduced oxidative stress promotes the formation of advanced glycation end products, PKC activation, hexosamine and polyol pathway activity $(18,24,25)$.

In our previous study, the FAM172A gene was cloned for the first time from human aortic tissues and its presence was first confirmed at the mRNA expression level (19). Our previous study also showed that the FAM172A gene was upregulated by high glucose levels in macrophages (19).

In the present study, we first wanted to know whether the FAM172A gene was expressed at the level of translation. We found that the transfection of the recombinant PDC315- 


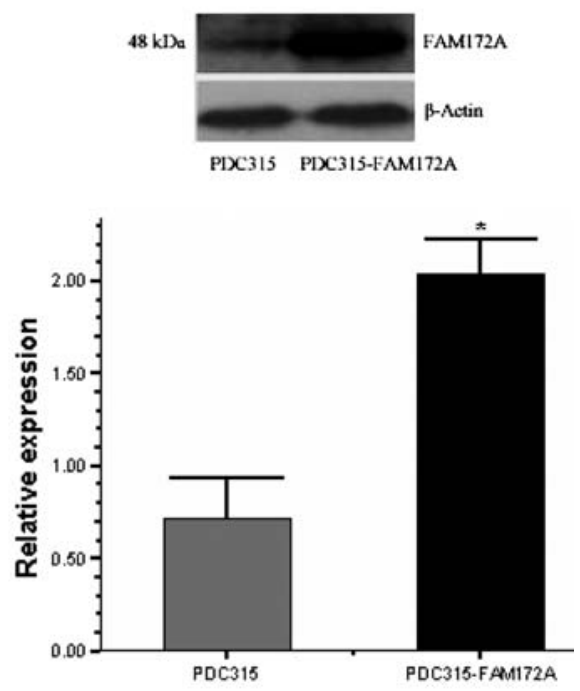

B

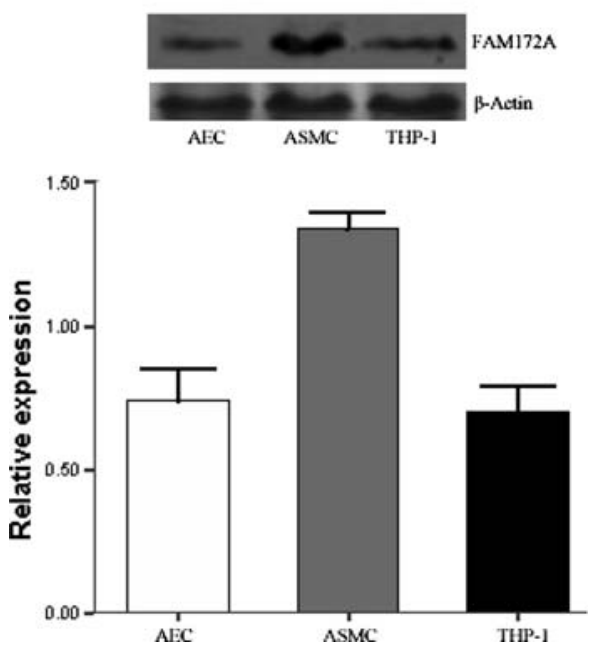

Figure 3. Identification and detection of the FAM172A protein in cells. (A) Identification of FAM172A at the level of translation. Western blotting demonstrated that compared to the transfection with the empty PDC315 vector, the expression of FAM172A in HEK293 cells was markedly increased after the transfection of recombinant PDC315-FAM172A plasmids into HEK293 cells. Lane 1, HEK293 cells transfected with empty PDC315 plasmids; 2, HEK293 cells transfected with recombinant PDC315-FAM172A plasmids. (B) Detection of FAM172A in vascular cells. Western blot analysis showed that the FAM172A protein was detectable in human aortic endothelial, human aortic smooth muscle cells and human THP-1-derived macrophages, and that the highest expression was observed in human aortic smooth muscle cells.
FAM172A vectors into the HEK293 cells led to the overexpression of the FAM172A gene in the HEK293 cells. This result suggests that the FAM172A gene could be expressed at the protein level. Therefore, we further confirmed the presence of the FAM172A gene at the level of translation.

Endothelial, vascular smooth muscle cells and macrophages are central to the initiation and progression of atherosclerosis. Our present results showed that the FAM172A protein was detected in human endothelial, human aortic smooth muscle cells and THP-1-derived macrophages, and the highest expression was found in human aortic smooth muscle cells. Given the fact that the expression of FAM172A was very high in human aortic smooth muscle cells, we further investigated the effects of high glucose levels on the FAM172A protein expression in aortic smooth muscle cells. Our study demonstrated that the FAM172A protein in human aortic smooth muscle cells was up-regulated by high glucose levels in a concentration-dependent and a time-course manner, which indicated that FAM172A was a glucose-induced gene and that the changes in expression of FAM172A could be one of the pathogenic mechanisms involved in glucose-mediated vascular damage.

The function of a protein is closely linked with both the cell location and the conserved domain. The FAM172A protein is a novel protein and its structure and function is still unknown. Bioinformatics analysis suggested that the FAM172A protein could be located in the nucleus and/or cytoplasm. Our experimental results demonstrated that HEK293 cells transfected with the pEGFP-N2-FAM172A construct showed weak green fluorescent product in the cytoplasm with strong nuclear positivity, which suggested that the FAM172A protein was located in both the nucleus and cytoplasm. This result was consistent with the bioinformatics prediction. However, by using the Conserved Domain Search service (CD-Search) $(26,27)$, an Arb2 conserved domain was detected in the FAM172A protein sequence. Arb2 is required for histone H3 Lys9 (H3-K9) methylation, heterochromatin assembly and siRNA generation (28). Histone H3 lysine methylation is generally associated with either gene activation or repression depending on the site of methylation $(29,30)$. The epigenetic modification, H3K9me3 is usually linked to gene repression (31). However, in certain cases, $\mathrm{H} 3 \mathrm{~K} 9 \mathrm{me} 3$ has been linked to gene activation (32). High glucose levels can promote inflammatory gene
A

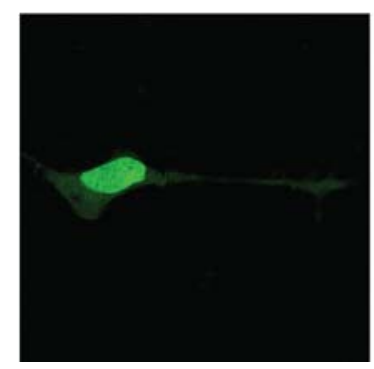

B

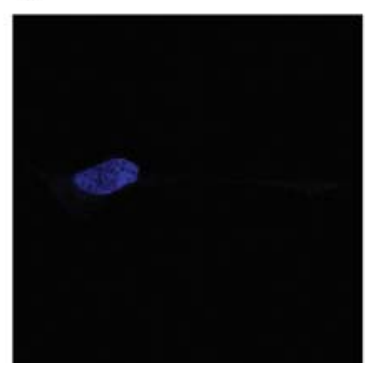

C

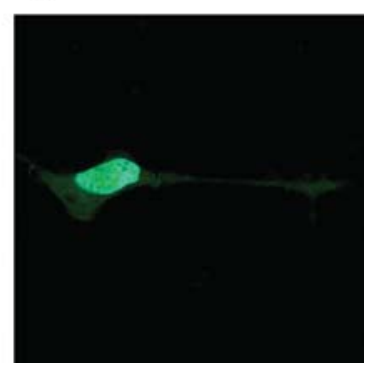

Figure 4. Subcellular localization of the FAM172A protein in HEK293 cells. Human HEK293 cells were transiently transfected with pEGFP-N2-FAM172A fusion constructs. (A) The FAM172A-GFP fusion protein (green) was localized predominantly in the nucleus. However, a weak green fluorescent signal was also detected in the cytoplasm. (B) DAPI staining analysis showed a blue fluorescent signal. (C) Converging the 2 images gave a yellow fluorescent pattern, which confirmed the co-location of the FAM172A-GFP and cell nuclei. 
A
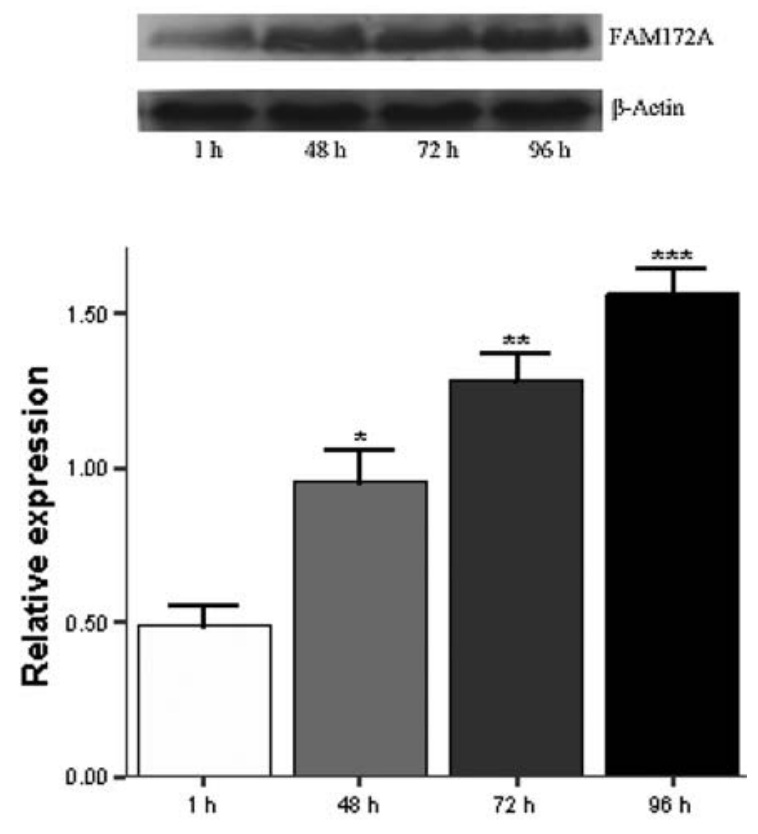

B

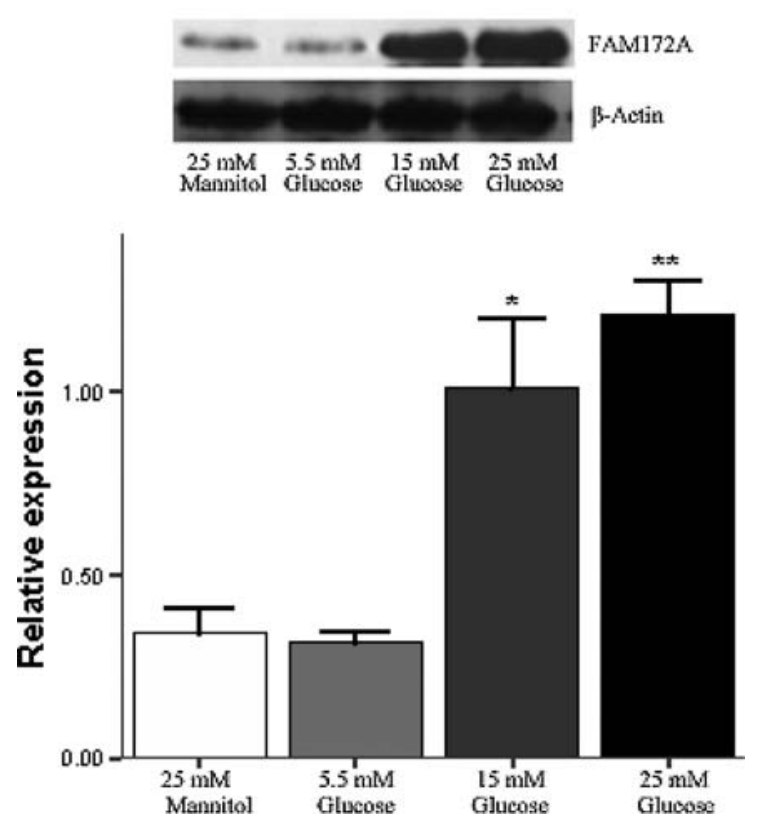

Figure 5 Regulation of the FAM172A protein expression by glucose. (A) The FAM172 protein expression in human aortic smooth muscle cells was up-regulated by high glucose in a time-course manner. Western blot analysis showed that the expression of the FAM172A protein in human aortic smooth muscle cells were augmented with high glucose concentrations in the 48-, 72 and 96-h culture times, respectively, when compared to the control group (1 h). (B) The FAM172 protein expression in human aortic smooth muscle cells was up-regulated by high glucose in a concentration-dependent manner. Western blotting demonstrated that compared to the $5.5 \mathrm{mM}$, the 15 and $25 \mathrm{mM}$ glucose concentrations significantly up-regulated the expression of the FAM172A protein in human aortic smooth muscle cells. Compared to $5.5 \mathrm{mM}$ glucose, $25 \mathrm{mM}$ mannitol had no effect on the expression of the FAM172A protein.

expression and lead to the dysregulation of $\mathrm{H} 3 \mathrm{~K} 9 \mathrm{me} 3$ in vascular smooth muscle cells (31). Studies have also demonstrated that $\mathrm{H} 3 \mathrm{~K} 9 \mathrm{me} 3$ plays an important role in the repression of inflammatory genes in vascular smooth muscle cells (31). Based on the above data, we hypothesized that the high glucose-responsive FAM172A gene could activate or repress genes associated with diabetic vascular damage, by modifying $\mathrm{H} 3 \mathrm{~K} 9 \mathrm{me}$. Further research should be undertaken in order to determine the effects of FAM172A on histone H3-K9 methylation.

According to the results from our current study on FAM172A, it is certain that high glucose levels increased the FAM172A protein expression in human aortic smooth muscle cells. We have presented evidence that FAM172A is a glucose-responsive gene, and that its expression is regulated by glucose levels. The relationship between FAM172A and diabetic vascular complications or diabetes is worth further investigation in the future.

In conclusion, the FAM172A protein was identified for the first time. We also expressed the recombinant FAM172A protein and generated the rabbit anti-FAM172A polyclonal antibody. By a combination of bioinformatics and confocal laser scanning microscopy, we determined that the FAM172A protein in HEK293 cells, was mainly located in the cell nuclei. We presented evidence that the FAM172A protein was detected in human aortic endothelial, human aortic smooth muscle cells and macrophages, and that the highest expression was observed in human aortic smooth muscle cells. We also presented evidence that the FAM172 protein expression was significantly increased by high glucose levels in human aortic smooth muscle cells. We speculate that FAM172A, as a novel protein, could be involved in the pathogenesis of high glucose-induced vascular damage.

\section{Acknowledgements}

This study was supported by grants from the Shanghai Key Laboratory of Diabetes Mellitus (no. 08DZ2230200) and the Shanghai Municipal Health Bureau (no. 2008070).

\section{References}

1. Haffner SM, Lehto S, Ronnemaa T, Pyorala K and Laakso M: Mortality from coronary heart disease in subjects with type 2 diabetes and in nondiabetic subjects with and without prior myocardial infarction. N Engl J Med 339: 229-234, 1998.

2. Beckman JA, Creager MA and Libby P: Diabetes and atherosclerosis: epidemiology, pathophysiology, and management. JAMA 287: 2570-2581, 2002.

3. Donnelly R and Davis KR: Type 2 diabetes and atherosclerosis. Diabetes Obes Metab 2: S21-S30, 2000.

4. Sowers JR, Epstein M and Frohlich ED: Diabetes, hypertension, and cardiovascular disease: an update. Hypertension 37: 1053-1059, 2001

5. Arauz-Pacheco C, Parrott MA and Raskin P: The treatment of hypertension in adult patients with diabetes. Diabetes Care 25: 134-147, 2002.

6. Haffner SM: Management of dyslipidemia in adults with diabetes. Diabetes Care 26: S83-S86, 2003.

7. Reusch JE and Draznin BB: Atherosclerosis in diabetes and insulin resistance. Diabetes Obes Metab 9: 455-463, 2007.

8. Gaede P, Lund-Andersen H, Parving HH and Pedersen O: Effect of a multifactorial intervention on mortality in type 2 diabetes. N Engl J Med 358: 580-591, 2008.

9. Leiter LA, Ceriello A, Davidson JA, Hanefeld M, Monnier L, Owens DR, Tajima N and Tuomilehto J: Postprandial glucose regulation: new data and new implications. Clin Ther 27: S42-S56, 2005.

10. Brunner EJ, Shipley MJ, Witte DR, Fuller JH and Marmot MG: Relation between blood glucose and coronary mortality over 33 years in the Whitehall Study. Diabetes Care 29: 26-31, 2006. 
11. O'Keefe JH and Bell DS: Postprandial hyperglycemia/ hyperlipidemia (postprandial dysmetabolism) is a cardiovascular risk factor. Am J Cardiol 100: 899-904, 2007.

12. Prince CT, Becker DJ, Costacou T, Miller RG and Orchard TJ: Changes in glycaemic control and risk of coronary artery disease in type 1 diabetes mellitus: findings from the Pittsburgh Epidemiology of Diabetes Complications Study (EDC). Diabetologia 50: 2280-2288, 2007.

13. Chait A and Bornfeldt KE: Diabetes and atherosclerosis: is there a role for hyperglycemia. J Lipid Res 50: S335-S339, 2009.

14. Nishio Y, Aiello LP and King GL: Glucose induced genes in bovine aortic smooth muscle cells identified by mRNA differential display. FASEB J 8: 103-106, 1994.

15. Shanmugam N, Reddy MA, Guha M and Natarajan R: High glucose-induced expression of proinflammatory cytokine and chemokine genes in monocytic cells. Diabetes 52: 1256-1264, 2003.

16. Chen S, Feng B, George B, Chakrabarti R, Chen M and Chakrabarti S: Transcriptional co-activator p300 regulates glucose induced gene expression in the endothelial cells. Am J Physiol Endocrinol Metab 298: E127-E137, 2010.

17. Wang X, Gong W, Liu Y, Yang Z, Zhou W, Wang M, Yang Z, Wen J and Hu R: Molecular cloning of a novel secreted peptide, INM02, and regulation of its expression by glucose. J Endocrinol 202: 355-364, 2009.

18. Nicolson GL: Metabolic syndrome and mitochondrial function: molecular replacement and antioxidant supplements to prevent membrane peroxidation and restore mitochondrial function. $\mathrm{J}$ Cell Biochem 100: 1352-1369, 2007.

19. Li LX, Tao Z, Dong XH, Liang WC, Yang ZH, Mou B, Bao YQ, Wang C, Jia WP and Hu RM: Molecular cloning of a novel gene, C5orf 21 gene and its roles in diabetic macroangiopathy. Zhonghua Yi Xue Za Zhi 89: 2574-2577, 2009.

20. Yu CS, Chen YC, Lu CH and Hwang JK: Prediction of protein subcellular localization. Proteins 64: 643-651, 2006.

21. Brownlee M: Biochemistry and molecular cell biology of diabetic complications. Nature 414: 813-820, 2001.

22. Aronson D: Hyperglycemia and the pathobiology of diabetic complications. Adv Cardiol 45: 1-16, 2008.
23. Brownlee M: The pathobiology of diabetic complications: a unifying mechanism. Diabetes 54: 1615-1625, 2005.

24. Du XL, Edelstein D, Rossetti L, Fantus IG, Goldberg H, Ziyadeh F, Wu J and Brownlee M: Hyperglycemia-induced mitochondrial superoxide overproduction activates the hexosamine pathway and induces plasminogen activator inhibitor-1 expression by increasing Sp1 glycosylation. Proc Natl Acad Sci USA 97: 12222-12226, 2000.

25. Ceriello A: New insights on oxidative stress and diabetic complications may lead to a 'causal' antioxidant therapy. Diabetes Care 26: 1589-1596, 2003.

26. Marchler-Bauer A, Anderson JB, Chitsaz F, Derbyshire MK, DeWeese-Scott C, Fong JH, Geer LY, Geer RC, Gonzales NR, Gwadz M, He S, Hurwitz DI, Jackson JD, Ke Z, Lanczycki CJ, Liebert CA, Liu C, Lu F, Lu S, Marchler GH, Mullokandov M, Song JS, Tasneem A, Thanki N, Yamashita RA, Zhang D, Zhang N and Bryant SH: CDD: specific functional annotation with the Conserved Domain Database. Nucleic Acids Res 37: D205-D210, 2009.

27. Marchler-Bauer Aand Bryant SH: CD-Search: protein domain annotations on the fly. Nucleic Acids Res 32: W327-W31, 2004.

28. Buker SM, Iida T, Buhler M, Villen J, Gygi SP, Nakayama J and Moazed D: Two different Argonaute complexes are required for siRNA generation and heterochromatin assembly in fission yeast. Nat Struct Mol Biol 14: 200-207, 2007.

29. Jenuwein T and Allis CD: Translating the histone code. Science 293: 1074-1080, 2001

30. Martin $\mathrm{C}$ and Zhang Y: The diverse functions of histone lysine methylation. Nat Rev Mol Cell Biol 6: 838-849, 2005.

31. Villeneuve LM, Reddy MA, Lanting LL, Wang M, Meng L and Natarajan R: Epigenetic histone H3 lysine 9 methylation in metabolic memory and inflammatory phenotype of vascular smooth muscle cells in diabetes. Proc Natl Acad Sci USA 105: 9047-9052, 2008.

32. Vakoc CR, Mandat SA, Olenchock BA and Blobel GA: Histone H3 lysine 9 methylation and HP1gamma are associated with transcription elongation through mammalian chromatin. Mol Cell 19: 381-391, 2005. 